\title{
Effect of elastic transfer in sub-barrier fusion between similar nuclei
}

\author{
M. Lozano \\ Departamento de Física Atómica y Nuclear, University of Sevilla, 41080 Sevilla, Spain
}

A. Vitturi

Dipartimento di Fisica "Galileo Galilei" and Istituto Nazionale di Fisica Nucleare, I-35131 Padova, Italy

(Received 31 March 1986)

\begin{abstract}
We study the effect of the elastic transfer process on sub-barrier fusion reactions between similar nuclei. The coupling to the elastic transfer channel is described in terms of a parity dependent potential, directly obtained from the transfer form factor. Within the simple barrier penetration model, we find that this effect can account for a considerable part of the sub-barrier enhancement of the ${ }^{16} \mathrm{O}+{ }^{18} \mathrm{O}$ fusion cross section over the ${ }^{16} \mathrm{O}+{ }^{16} \mathrm{O}$ case.
\end{abstract}

The effect of the transfer process in elastic scattering between similar nuclei is well known. ${ }^{1}$ In fact, the interference between the truly elastic process and the transfer of particles can be considered as the mechanism responsible for the oscillations which are present in the backward angle elastic cross sections. Such an effect can be incorporated in the standard optical model framework by introducing a parity dependent contribution $(-)^{l} \Delta U_{\mathrm{ET}}(r)$ to the ion-ion potential. As shown, for example, in Ref. 2 , this potential can be easily expressed in terms of the form factor associated with the transfer process.

The aim of this paper is to see whether the same process can lead to appreciable effects in the sub-barrier fusion reactions. Because of the elastic transfer process, even and odd partial waves will penetrate barriers of different heights $U\left(r_{B}\right) \pm \Delta U_{\mathrm{ET}}\left(r_{B}\right)$. Although the two opposite contributions to the potential have equal magnitude and the elastic transfer is obviously a $Q=0$ transition, a net increase in the cross section can be expected due to its exponential-type dependence on the barrier energy.

For a quantitative analysis we took the case of the subbarrier fusion process between oxygen nuclei, ${ }^{3}$ which shows a clear isotopic difference. The measured enhancement factors of the ${ }^{16} \mathrm{O}+{ }^{18} \mathrm{O}$ fusion cross sections over the corresponding ${ }^{16} \mathrm{O}+{ }^{16} \mathrm{O}$ cases, shown in Fig. 1 as a function of the center of mass energy, can in fact only partially be attributed to the scaling of the radius due to the change in the mass number. We have evaluated fusion cross section using the simple one-dimensional barrier penetration model in the Hill and Wheeler approximation. The standard empirical potential by Christensen and Winther $^{4}$ in the Woods-Saxon version has been used for the ion-ion potential to determine radius, height, and curvature of the barrier. For the ${ }^{16} \mathrm{O}+{ }^{18} \mathrm{O}$ case, the even-odd partial wave difference in the potential has been taken into account by evaluating the fusion cross section according to

$$
\begin{aligned}
\sigma_{\text {fus }}(E)=\frac{1}{2}\{ & \sigma_{\text {fus }}\left[U\left(r_{B}\right)+\Delta U_{\mathrm{ET}}\left(r_{B}\right), E\right] \\
& \left.+\sigma_{\text {fus }}\left[U\left(r_{B}\right)-\Delta U_{\mathrm{ET}}\left(r_{B}\right), E\right]\right\} .
\end{aligned}
$$

For the parity dependent potential $\Delta U_{\mathrm{ET}}(r)$ we have followed the simple prescription of Ref. 2, based on the macroscopic approach to two-particle transfer processes, which gives the expression

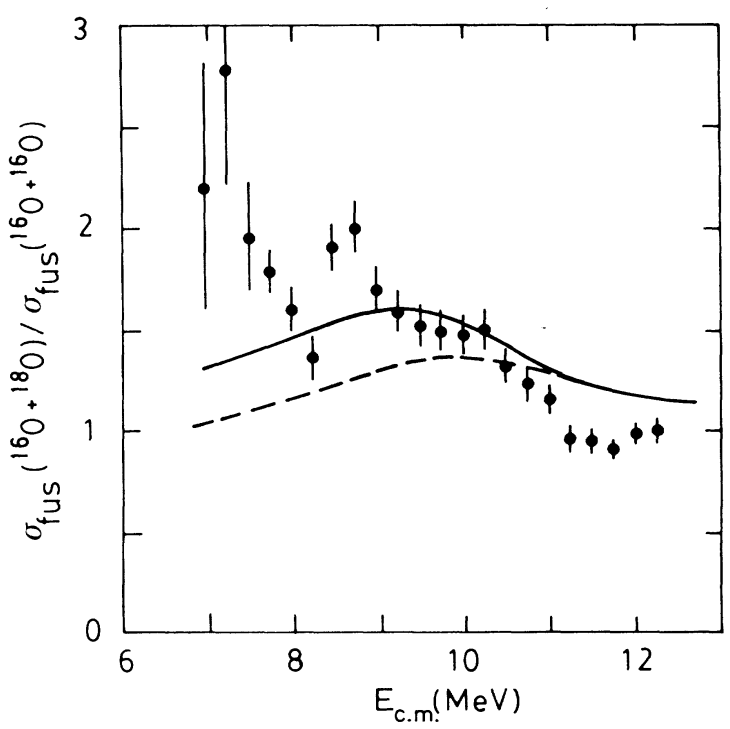

FIG. 1. Enhancement factors of the ${ }^{16} \mathrm{O}+{ }^{18} \mathrm{O}$ fusion cross section over the ${ }^{16} \mathrm{O}+{ }^{16} \mathrm{O}$ cross section. The experimental data are taken from Ref. 3. The dashed line gives the prediction obtained by simply scaling the radius of the potential according to the mass number. The full line is obtained by including the parity-dependent potential due to the elastic transfer process. 


$$
\Delta U_{\mathrm{ET}}(r)=\left(\beta_{p} R / 3 A \sqrt{4 \pi}\right) d U(r) / d r .
$$

With the value $\beta_{p}=16$ for the pair-deformation parameter, this parity dependent term leads to a fairly good description of the oscillations in the backward angle ${ }^{16} \mathrm{O}+{ }^{18} \mathrm{O}$ elastic scattering. ${ }^{2}$

The resulting fusion enhancement factors (solid line) are compared with the experimental values in Fig. 1. We also show, as a dashed line, the contribution to the enhancement originated by the number of mass dependence of the ion-ion potential. As is apparent from the figure, for energies below the barrier $(10.2 \mathrm{MeV})$ the elastic transfer leads to an appreciable increase of the fusion cross section of the order of $30 \%$, while the enhancement tends to vanish as the bombarding energy increases over the barrier.

The shape of the calculated cross sections (as well as the experimental one) shows a bump at the energy around 9-10 MeV. This behavior can be explained in a simple way. If one takes into account the $S$ wave, the fusion cross section is expressed as

$$
\sigma=\frac{\pi}{k^{2}} T=\frac{\pi \hbar^{2}}{2 \mu} \frac{1}{E} T
$$

where $T$ is the transmission coefficient for the $S$ wave. In the transient energy regions $T$ increases from 0 to 1 . The transient region is characterized simply by the potential barrier height, which is roughly determined by the product of the charges as well as the radius. The latter is slightly different between the two systems. Thus, the ratio

$$
R=\frac{\mu_{2} T_{1}}{\mu_{1} T_{2}}
$$

has a bump in the transient energy region.

In Ref. 5 the isotopic difference in the oxygen fusion reactions was fairly well interpreted within a coupledchannel model and mainly ascribed to the coupling to the low $2^{+}$state in ${ }^{18} \mathrm{O}$. Although we do not think that the elastic transfer process can be viewed as an alternative mechanism completely responsible for the enhancement, we can, however, conclude from our calculation that its contribution is not at all negligible and should therefore be taken into account.

One of us (A.V.) would like to thank the Departamento de Fisica Atómica y Nuclear of the University of Sevilla for kind hospitality. This work was partially supported by the Spanish Comisión Asesora de Investigación Científica y Técnica, under Contract no. 2868/83.
${ }^{1}$ W. von Oertzen and H. G. Bohlen, Phys. Rep. 19C, 1 (1975).

${ }^{2}$ C. H. Dasso and A. Vitturi (unpublished).

${ }^{3}$ J. Thomas, Y. T. Chen, S. Hinds, K. Langanke, D. Meredith, M. Olson, and C. A. Barnes, Phys. Rev. C 31, 1980 (1985).
${ }^{4}$ P. R. Christensen and A. Winther, Phys. Lett. 65B, 19 (1976).

5J. Q. Wu, G. Bertsch, and A. B. Balantekin, Phys. Rev. C 32, 1432 (1985). 